JOURNAL OF BUSINESS

and entrepreneurial

studies

\title{
Estratégias de marketing para operadoras turística en la parroquia Posorja Provincia del Guayas
}

\section{Marketing strategies for tour operators in the parish Posorja Province of Guayas}

Ing. Enith Rosamelia López Franco Mgs. Universidad de Guayaquil Guayaquil-Ecuador enith_1f@ug.edu.ec ORCID https://orcid.org/0000-0001-9373-3729

Ing. Stalin Flavio Fiallos Castillo Mgs. Instituto Tecnológico del Valle De Oaxaca

Oaxaca-México stafica@hotmail.com ORCID https://orcid.org/0000-0001-6047-1080

Recibido 7 marzo 2017 - Aceptado 17 julio- 2017 



\section{Resumen}

La investigación se realizó en la parroquia Posorja provincia del Guayas, cuyo propósito es el desarrollo de estrategias de marketing para el fortalecimiento de la actividad turística en sus operadoras Fue necesario la realización de un diagnóstico situacional del turismo, para verificar las fortalezas, oportunidades, debilidades y amenazas; se empleó un estudio de mercado que permitió identificar deseos $y$ preferencias del cliente y su posición respecto a la competencia, planteando estrategias de posicionamiento. Este trabajo se justificó porque contribuye con el desarrollo fundamental de los recursos, permitiendo de esta forma tomar las medidas preventivas, garantizando un control ordenado $\mathrm{y}$ específico para el desarrollo de la actividad turística, por tal motivo se beneficiará no solo la operadora turística, sino también al sector en particular. Se sustentó en las teorías de la planeación de estrategias de marketing y el turismo establecidas en el marco teórico, la investigación se aborda de acuerdo a la metodología deductiva e inductiva, determinando el estudio descriptivo y utilizando las técnicas de la observación, la entrevista y la encuesta. La población fue tomada en diferentes hoteles de Playas y Posorja con un total de 538 turistas, la muestra probabilística fue de 230 personas, se empleó una encuesta a los turistas formada por 10 preguntas y una entrevista al presidente del GAD parroquial de Posorja, obtenida la información se llegó a la discusión de los resultados. En el desarrollo de la propuesta se establecieron estrategias y tácticas, para de esta forma realizar campañas de promoción y publicidad, logrando el posicionamiento de la empresa y su marca; dentro de los impactos de la propuesta se tomó en cuenta lo económico, lo social y lo ambiental. Finalizando con las conclusiones y recomendaciones que amerita el desarrollo de este trabajo de titulación.

Palabra clave: Turismo, Operadoras Turísticas, Promoción , Estrategias, Marketing

\section{Abstract}

The research was carried out in the parish of Posorja province of Guayas, whose purpose is the development of marketing strategies to strengthen tourism activity in its operators It was necessary to carry out a situational diagnosis of tourism, to verify the strengths, opportunities, weaknesses And threats; A market study was used to identify client's wishes and preferences and their position regarding the competition, proposing positioning strategies. This work was justified because it contributes to the fundamental development of the resources, allowing in this way to take the preventive measures, guaranteeing an orderly and specific control for the development of the tourist activity, for that reason will benefit not only the tour operator, but also To the sector in particular. It was based on theories of the planning of marketing strategies and tourism 
established in the theoretical framework, the research is approached according to the deductive and inductive methodology, determining the descriptive study and using the techniques of observation, interview and poll. The population was taken in different hotels in Playas and Posorja with a total of 538 tourists, the probabilistic sample was 230 people, was used a survey to the tourists formed by 10 questions and an interview to the president of the parochial GAD of Posorja, obtained the Information was reached the discussion of the results. In the development of the proposal strategies and tactics were established, in order to carry out promotional and publicity campaigns, achieving the positioning of the company and its brand; Within the impacts of the proposal took into account the economic, social and environmental. Finalizing with the conclusions and recommendations that merit the development of this titling work.

Key word: Tourism, Tour Operators, Promotion, Strategies, Marketing

\section{Introducción}

En la actualidad, el turismo ha dado un crecimiento muy acelerado, debido a la gran demanda que cada vez se convierte más exigente y competitiva, transformándose en un fenómeno social. Es por esta razón que varias empresas, llámese así, empiezan empíricamente con el afán de ofrecer un servicio que le signifique un modo de ingreso económico; pero este en muchas ocasiones es poco rentable y no muy satisfactorio, debido a la falta de conocimiento en el negocio incursionado.

La elaboración de un plan estratégico de marketing marca un rol muy significativo al momento de tomar decisiones, permitirá analizar todos los factores necesarios, sus fortalezas, oportunidades, debilidades y amenazas en la que se vea involucrada la compañía de turismo. Esta es una forma muy eficaz y eficiente para asegurar a largo plazo la permanencia de la misma.

Uno de los principales atractivos de la parroquia Posorja es el avistamiento de los delfines nariz de botella, que localmente son llamados bufeos. Por tal motivo, un grupo de emprendedores observaron esta atracción tan singular, que provocaban estos amigables mamíferos, cuando navegaban en sus embarcaciones pesqueras; aprovechando esta oportunidad, decidieron crear empíricamente una pequeña empresa. Esta inició el 25 de enero del 2005 como una comisión turística. Después de dos años de emprendimiento se transformó en la Asociación "Los Punaes", en la cual se brindaba el servicio de transportación marítima, de carga y en ese entonces la de turismo. Debido a las exigencias y normativas del Ministerio de Turismo, se les indicó a los miembros de la asociación que, para brindar el servicio turístico, ellos debían constituirse legalmente como una operadora o agencia de turismo. Por tales circunstancias desde el 23 de agosto del 2011, se creó bajo la razón social de "Compañía de turismo El Paraíso de 
Puná S.A. (T.P.P.)", con el RUC \# 0992806885001, la cual está ubicada en el malecón de la parroquia Posorja, junto al parque turístico de la localidad y diagonal al hotel que lleva el mismo nombre de la parroquia.

La parroquia Posorja no es reconocida como un sector turístico. La falta de capacitación y de asesoría técnica, ha hecho que exista una sola operadora que brinde este servicio. Esto conlleva a que los miembros de la operadora no tengan un control de sus ingresos y gastos, puesto que lo generado en cada salida turística es para beneficio propio del dueño de la embarcación.

En los actuales momentos no existe una buena infraestructura, inversión y peor aún publicidad, que ayude a los miembros de las operadoras turísticas darse a conocer a nivel local y nacional. Factores como estos han hecho que muchas personas, incluyendo a los locales, no conozcan del atractivo en particular, puesto que no existe un plan de marketing que ayude a impulsar el desarrollo turístico en el sector, para lograr una mayor afluencia de visitantes, mejorando la economía de la parroquia. El acuerdo ministerial No. 20140007, dado el 03 de febrero del 2014, menciona que la constitución de la República en sus artículos 24 y 66 reconoce y garantiza a las personas el derecho a una vida digna que asegure, entre otros, el descanso y ocio, como el derecho a esparcimiento, los cuales pueden ser ejercidos a través de las distintas modalidades turísticas establecidas conforme a la ley.
El turismo es declarado por el gobierno nacional como una política de estado, encaminada a la consecución del buen vivir a través de la generación de empleo, cadenas productivas, divisas, redistribución de la riqueza e inclusión social.

Según la Ministra de Turismo Sandra Naranjo, indicó en un reportaje publicado el 04/06/2015 por el diario Ecuadorinmediato.com, que "El turismo en el Ecuador está generando millones de dólares, la industria del ocio generó 1.487 millones de dólares en lo que fue del año 2014 y se ha convertido en la tercera fuente de ingresos no petroleros después del camarón y los servicios empresariales".

La finalidad de este trabajo de titulación es de dar a conocer la importancia de elaborar un plan estratégico de marketing, para las operadoras turísticas, proponiendo estrategias que ayude a mejorar el proceso administrativo de la misma, con el objetivo de incentivar y promover el potencial turístico que existe en la localidad de Posorja.

Existen diversas alternativas turísticas, que no están siendo explotadas de forma correcta debido a la falta de creatividad e innovación, por tal razón el desarrollo de este plan estratégico de marketing se convierte en un factor fundamental para ofrecer un producto turístico de alta calidad, que busca atraer la afluencia de turistas e incrementar la generación de divisas que en ella demande.

El presente trabajo de titulación se lo considera factible porque permitirá tomar las medidas preventivas y ayudará a garantizar mediante el plan estratégico 
de marketing, un control ordenado y específico para el desarrollo de la actividad turística en el sector, permitiendo a los habitantes obtener una nueva plaza de empleo y otra fuente generadora de ingresos.

$\mathrm{Su}$ objetivo principal es de desarrollar estrategias de marketing para el fortalecimiento de la actividad turística en sus Operadoras de la Parroquia Posorja, Provincia del Guayas.

\section{Materiales y métodos}

Establece la comprensión y descripción de las etapas o fases necesarias para cumplir con el desarrollo de la investigación y el tipo de estudio del cual se trata.

Mediante este proceso de investigación en conjunto con las técnicas y procedimientos cuyo propósito fundamental fue el de implementar procesos de recolección, clasificación y validación de datos, de acuerdo a las experiencias y conocimientos obtenidos de la realidad en que se encuentra las operadoras turísticas, basándonos en sus actividades turísticas, se pudo construir el proceso investigativo de este trabajo, sustentado en la investigación bibliográfica documental, en la investigación de campo como la observación, la entrevista y la encuesta, que se constituirá como un aliciente para llegar a las conclusiones y recomendaciones de este trabajo.

El método de la investigación es el conjunto de procedimientos lógicos a través de los cuales se plantean los problemas científicos, esto es de una forma estructurada o sistemática para dar solución a los problemas y llegar a los objetivos fijados.

El presente estudio es de carácter, deductivo e inductivo que permite palpar la realidad del hecho a investigar $\mathrm{y}$ establecer las variables de causas y efectos, sea de manera cuantitativa o cualitativa, contribuyendo con el objetivo y el desarrollo del plan estratégico de marketing turístico.

Se realiza un análisis de las ventajas y desventajas de la misma utilizando la investigación de campo, la que permitirá realizar la recolección de información en el lugar de los hechos.

Por medio de un estudio estructurado y sistemático se emplea la investigación descriptiva con el fin de mejorar las expectativas de las operadoras turísticas, convirtiéndola en una de las principales en la zona.

Se la realiza por medio del problema estructurado en la investigación, en la que consiste como la falta de promoción y publicidad a las operadoras turísticas. Esto nos lleva a deducir el bajo posicionamiento que tiene en el sector y a detectar que los turistas desconocen del tema.

La inducción es una argumentación, por tal razón conlleva un análisis ordenado, coherente y lógico del problema de investigación, tomando como referencia, antecedentes verdaderos.

Este método es un proceso analítico y sintético en el que intervino el estudio de la causa y el efecto del problema, en tal circunstancia, ayudó a obtener las conclusiones a partir de los resultados durante la aplicación del mismo. 
La inducción, podrá llegar a conclusiones cuyo contenido es el análisis del problema de investigación.

Constituye en un proceso sistemático de recolección, tratamiento, análisis y presentación de datos, que se realiza en el lugar mismo donde ocurren los fenómenos sujetos a estudio. Estudia los fenómenos sociales en su ambiente natural, se apoya en informaciones que provienen de entrevistas, cuestionarios, encuestas y observaciones.

En consideración a lo expuesto, este trabajo se lo desarrolló en el lugar en que se producen los acontecimientos, realizados por la operadora turística "El Paraíso de Puná (T.P.P.)", con el propósito de descubrir, entender $\mathrm{y}$ explicar su naturaleza, para ello se direccionó hacia los miembros directivos de la operadora y de esta forma recolectar toda la información necesaria que ellos pudieron proporcionar, estableciendo así el aporte que brindará este trabajo, posterior a esto se dio comienzo la realización del recorrido turístico, partiendo de las instalaciones de la operadora que está ubicada en el malecón de la parroquia Posorja, junto al parque turístico de la localidad $\mathrm{y}$ diagonal al hotel que lleva el mismo nombre de la parroquia.

Incursionando en la aventura que significa realizar esta travesía marítima y visitando aquellos lugares a promocionar en el paquete turístico completo, como lo es el avistamiento de los delfines nariz de botella, observación de aves acuáticas, la Isla de los Farallones, la isla de los pájaros ubicada en el centro de Refugio de Vida Silvestre Manglares El Morro
"REVISMEN", el cual goza de un ecosistema estuario de isla de manglar y posee la majestuosa e inmensa población de fragatas de toda la costa ecuatoriana, visita a la isla Puná específicamente el sector de la comuna Subida Alta que ofrece un ambiente muy particular $\mathrm{y}$ exclusivo.

Esto se efectuó con la finalidad de recolectar toda la información necesaria para realizar este trabajo de titulación que servirá como un aporte y beneficio para la operadora y la comunidad posorjeña.

Es la revisión bibliográfica del tema, para conocer el estado del problema a investigar. Se fundamenta en la revisión sistemática, rigurosa y profunda del material, de acuerdo al análisis de la relación de dos o más variables, cuando el investigador escoge este tipo de estudio, utiliza documentos, recolecta toda la información, selecciona, analiza y presenta los resultados más afines.

Para la realización y desarrollo de este trabajo investigativo, se recopilaron datos de diferentes fuentes como lo son: libros, revistas, periódicos, páginas web, tesis educativas, profundizando de esta forma los aspectos teóricos que permitieron la solución al problema planteado en las operadoras turísticas.

Tiene como objetivo central lograr la descripción o caracterización de un evento de estudio dentro de un contexto, como fin particular describe cosas como las actitudes de los consumidores ante una marca y sus opiniones sobre el lanzamiento de un nuevo producto.

Por medio de esta investigación, se permitió detallar las características más 
importantes y relevantes del objeto de estudio, cuyo propósito fue la delimitación de los hechos, reuniendo argumentos específicos y fundamentales que conforman el problema de la investigación que presenta las operadoras turísticas, con la finalidad de proponer mejoras para impulsar el desarrollo y crecimiento turístico, incrementando la afluencia de visitantes para beneficio de la operadora.

Es el conjunto de instrumentos y medios a través del cual se efectúa el método y solo se aplica a una ciencia. Las técnicas de investigación son indispensables en el proceso de la investigación científica ya que integra la estructura por medio de la cual se organiza la investigación.

Existen varias técnicas para escoger la información como la observación directa o indirecta, entrevistas, encuestas, cuestionarios y sondeos de opiniones, por tal razón es de mucha importancia que la persona o investigador, determine cuál será el procedimiento que mejor se ajuste para la codificación y tabulación. De acuerdo a estas determinaciones, las técnicas que se eligieron y que mejor se ajustaron para el desarrollo de un plan de marketing en las operadoras turísticas, fue la observación, la entrevista y la encuesta, las cuales fueron utilizadas en el tratamiento de la información.

\section{Resultados}

El análisis e interpretación de la información en el proceso investigativo, depende del enfoque y del tipo de investigación que se haya seleccionado, como también de los objetivos planteados.
Para desarrollar el análisis de estudio y obtener los resultados requeridos cuyo propósito fue el de elaborar un plan estratégico de marketing para las operadoras turísticas, se utilizó la técnica de la encuesta, estructurándose un cuestionario de 10 preguntas cerradas que fueron dirigidas a los turistas, entre el Cantón Playas, Data de Posorja y la Parroquia Posorja durante un fin de semana, para conocer los deseos y preferencias al momento de elegir un servicio o producto turístico que satisfaga sus necesidades.

Siguiendo paso a paso la metodología establecida en función al trabajo de campo realizado, se evaluó de forma ordenada cada una de las interrogantes planteadas por la encuesta para el cumplimiento de los resultados que ayudaron a resolver la problemática que presenta las operadoras turísticas.

Los resultados obtenidos en la aplicación de la encuesta, fueron codificados y tabulados mediante datos estadísticos y descriptivos, para su procesamiento en cuadros, frecuencias y porcentajes.

A continuación se presenta en tablas y gráficos tabulados, la interpretación de cada una de las preguntas efectuadas en la encuesta, para una mayor visualización y comprensión de la misma.

\section{Discusión}

De acuerdo al análisis de la información referente a los datos tabulados, se obtuvo el resultado de la investigación de campo que a continuación se detalla: 
Se establecieron criterios de comportamiento y exigencias sobre las personas de acuerdo a un producto que satisfaga todas sus necesidades, y que la mejor forma de hacer turismo es en grupos como una medida de integración de amigos o familiares.

Se determinó en base al análisis de la encuesta que la mejor época para incluir y promocionar un paquete turístico es en el de temporada playera, y que los servicios más exigidos por los turistas es el de hospedaje, alimentación $y$ transporte, opciones que servirán para tomar en cuenta a la hora de brindar un servicio que satisfaga todas las expectativas y exigencias de los clientes. Es muy importante observar y analizar que las personas buscan el precio que mejor se adapte a su economía, siempre $y$ cuando el servicio que se les ofrezca tenga todas las garantías y seguridades necesarias.

Para el cliente todo debe estar en óptimas condiciones, como hospedajes y los diferentes servicios que se les ofrezcan, deben ser de calidad porque al final el cliente es el que paga y siempre tiene la razón.

Se estableció que para promocionar la operadora turística se deben realizar promociones a través de los diferentes medios de comunicación específicamente las redes sociales de moda.

En relación a lo mencionado, es importante emplear un plan de marketing que ayude a establecer estrategias y tácticas que ayude a resolver el problema de la falta de conocimiento turístico de las personas con relación a las operadoras turísticas de la parroquia Posorja, logrando así un incremento en la economía y la generación de nuevas plazas de empleo.

\section{Conclusión}

La parroquia Posorja posee diversos atractivos turísticos naturales, Así mismo la falta de conocimiento turístico, organización y de planificación impide el desarrollo adecuado de sus actividades.

Con la implementación de este trabajo de titulación, que corresponde a la planificación estratégica de marketing, se establece la presencia de publicidad y promoción para las operadoras turísticas de la parroquia Posorja, mejorando la atención al cliente y todo lo que respecta al servicio brindado por la operadora.

Se pudo definir la necesidad de implementar y desarrollar mecanismos de estrategias y tácticas para promocionar y posicionar a nivel turístico de las operadoras turísticas de la parroquia Posorja, promoviendo los atractivos y encantos naturales que posee la parroquia Posorja.

La presencia del plan estratégico de marketing, se constituye en un aporte al desarrollo turístico garantizando beneficios que favorecen a las operadoras turísticas de la parroquia Posorja, así como también a la localidad parroquial.

El planeamiento estratégico de marketing se convierte en un pilar fundamental para el aporte de soluciones, convirtiéndose en un instrumento para las mejoras 
administrativas de las operadoras turísticas de la parroquia Posorja. Así como también permitirá que los habitantes, valoren, preserven $\mathrm{y}$ conserven los recursos naturales y medios ambientales que posee la parroquia Posorja.

\section{Referencias}

Capriotti, P. (2013). Planificación Estratégica de la Imagen Corporativa. En P. Capriotti, Planificación Estratégica de la Imagen Corporativa (pág. 75). Málaga, España: Editorial Ariel / ISBN: 84-344-1275-6.

Gaxiola, Jesús. (Abril-Junio de 2013). 5 Pasos para elaborar un plan estratégico. Sonorait Innovación y Tecnología, 22; 23; 24.

Jesus Palencia; Huber García; Adriana Moreno; Carlos Rairan; Pablo Prado; Paula Rodriguez. (2013). Planeación Estratégica Diseñando la estrategia ganadora. Medellín: Proyecto Editorial San Matías / ISBN: 978958-57832-2-5.

KOTLER, PHILIP Y ARMSTRONG, GARY. (2012). Marketing Decimocuarta Edición. México: PEARSON EDUCACIÓN / ISBN: 978-607-32-1420-9.

KOTLER, PHILIP y ARMSTRONG, GARY. (2013). Fundamentos de marketing - Decimoprimera edición. México: PEARSON
EDUCACIÓN / ISBN: 978-607-321722-4 / ISBN: 978-607-32-17231 /ISBN: 978-607-32-1724-8.

KOTLER, PHILIP Y KELLER, KEVIN. (2012). Dirección de Marketing Decimocuarta edición. México: PEARSON EDUCACIÓN / ISBN: 978-607-32-1245-8 - ISBN: 978607-32-1250-2 - ISBN: 978-60732-1244-1.

O. C. Ferrell, Michael D. Hartline. (2012). Estrategia de marketing, Quinta edición. México, D.F.: (C) D.R. 2012 por Cengage Learning Editores, S.A. de C.V. /ISBN: 9780-538-46738-4 - ISBN: 978-607481-824-6.

Organización Mundial del Turismo. (17 de Abril de 2015). World Tourism Organization - UNWTO. Obtenido de World Tourism Organization - UNWTO: http://www2.unwto.org/es

Wallace Stettinius, D. Robley Wood Jr., Jacqueline L. Doyle, John L. Colley Jr. (2009). Plan de negocio Cómo diseñarlo e implmentarlo. (2. B. Profit Editorial, Ed., \& A. G. Bertrán, Trad.) Barcelona: The McGraw-Hill Companies, Inc. Bresca Editorial, S.L. 2009 / ISBN: 978-84-936084-2-2. 